\section{Multi-Spectral Imaging and Linear Unmixing Add a Whole New Dimension to Laser Scanning Fluorescence Microscopy}

\author{
M.E. Dickinson, G. Bearman, S. Tille', \\ R. Lansford, and S.E. Fraser \\ California Institute of Technology, Pasadena, CA, \\ USA, and ${ }^{1}$ Carl Zeiss Jena GmbH, Jena, Germany
}

Multicolor fluorescence microscopy has become a popular way to discriminate between multiple proteins, organelles, or functions in a single cell or animal and can be used to approximate the physical relationships between individual proteins within the cell, for instance, by using fluorescence resonance energy transfer (FRET). These techniques have become invaluable to all biologists from cytogeneticists to zoologists. Resolving multiple, co-localized chromosome probes and individual fluorescent species in corals share the common problem of spectral overlap. Even in simple systems where two or three fluorochromes are used, spectral overlap or cross-talk can be difficult to eliminate, limiting the ability to distinguish one signal from another with any confidence. However, this problem is not unique to the biologist; geologists who use remote sensing techniques have been struggling with the problem for a long time. In fact, their problems are actually worse because many geological spectra are very similar and the number of possible spectral classes is much larger than the number of fluorescent probes in a biology experiment. Nonetheless, these issues have been tackled successfully, and biologists have recently begun to employ these methods to decipher multiple spectral signatures within cells and organisms, a process known as emission fingerprinting. Here, we review some approaches that have been used to enhance spectral resolution in fluorescence microscopy.

\section{GLASS FILTERS ONLY SCRATCH THE SURFACE OF MULTICOLOR DETECTION}

Classically, color separation of fluorescence emission has been achieved by using a set of optical bandpass filters that select different parts of the emission signals for every image channel. Such an approach is ideal if the emission spectra of the different fluorochromes do not overlap in the selected wavelength ranges. In practice, the dyes available are not always distinct enough to choose filter combinations that will eliminate overlap, and a complete separation of emission signals often results in a severe reduction in signal intensity. Some confocal laser scanning microscopes offer another option. For instance, multi-tracking uses electronic control of the scanners and the laser lines to switch the excitation wavelength between scans, either line-wise or frame-wise. This se- quential method avoids emission cross-talk by limiting the simultaneous emission of both fluorochromes. This approach is ideal for a number of applications in confocal microscopy; however, it does not help to separate emissions stimulated by the same excitation laser line. This holds true especially for multi-photon microscopy. Furthermore, as new fluorochromes are developed, they may not conform to the requirements of standard filter sets. As an example, many fluorochromes, such as the fluorescent proteins, have excitation and emission curves that closely overlap and will never be separated by purely physical means (Figure 1a). Thus, progress in fluorescence imaging requires flexibility.

\section{RECORDING THE COMPLETE EMISSION SPECTRA: LAMBDA STACK ACQUISITION}

The true challenge in multicolor imaging is the ability to separate the fluorescence contribution from multiple dyes that overlap spectrally and spatially. For instance, if cells are labeled with GFP in the cytoplasm and YFP in the cytoplasm and the nucleus, can these two signals be separated and assigned colors that indicate the distribution of one dye versus another? Currently, there is no filter combination or multitracking method that can be used for this; however, if a spectral image cube or lambda stack is generated, then these spectra can be separated using mathematical algorithms.

A lambda stack is a stack of $x-y$ images that sample emission data from a series of small wavelength bands (usually 5-12 nm depending on the device). Lambda stacks can be considered in much the same way as a time series or z-dimension series. The $x-y$ data can be viewed along the wavelength axis to determine how the intensity of pixels in the image change because of the amount of signal at different emission bands. Therefore, the emission spectra of a particular dye can be revealed by plotting the pixel intensity versus the center wavelength of each emission band (Figure 1b). The quality of the spectra obtained depends largely on the number of images collected at distinct wavelength bands, the size of the wavelength bands, and the sensitivity and reproducibility of the device used to collect the data.

Spectral imagers have emerged as new tools for acquiring lambda stacks using both wide-field and laser scanning microscopy (3). For instance, an interferometric device that uses a combination of spectroscopy, CCD imaging, and conventional microscopy (model SD-200; Applied Spectral Imaging, Migdal Haemek, Israel) $(1,4,6)$ has been used extensively for chromosome karyotyping. Other manufacturers such as Cambridge Research and Instrumentation (Cambridge, MA, USA) and Microcosm (Gaithersburg, MD, USA) have followed suit, developing other spectral imaging devices.

For confocal and two-photon laser scanning microscopy, liquid crystal tunable filters (LCTF) and acousto-optic tunable filters (AOTF) have been used to generate lambda stacks $(2,5)$. In both examples, the tunable filter is inserted in front of the photomultiplier tube (PMT) and selects a wavelength for that image. The LCTF can be tuned by electronically changing the conformation of the liquid crystal media, while AOTFs are tuned by changing the frequency of the resonating 
crystal; thus, $x-y$ scans can be made across a series of wavelengths generating a lambda stack. Using a combination of the hardware mentioned above and mathematical analysis, multiple probes can be distinguished in a mixed sample.

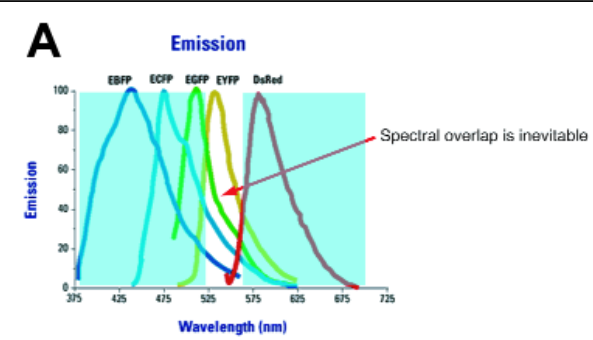

Original by www.clontech.com
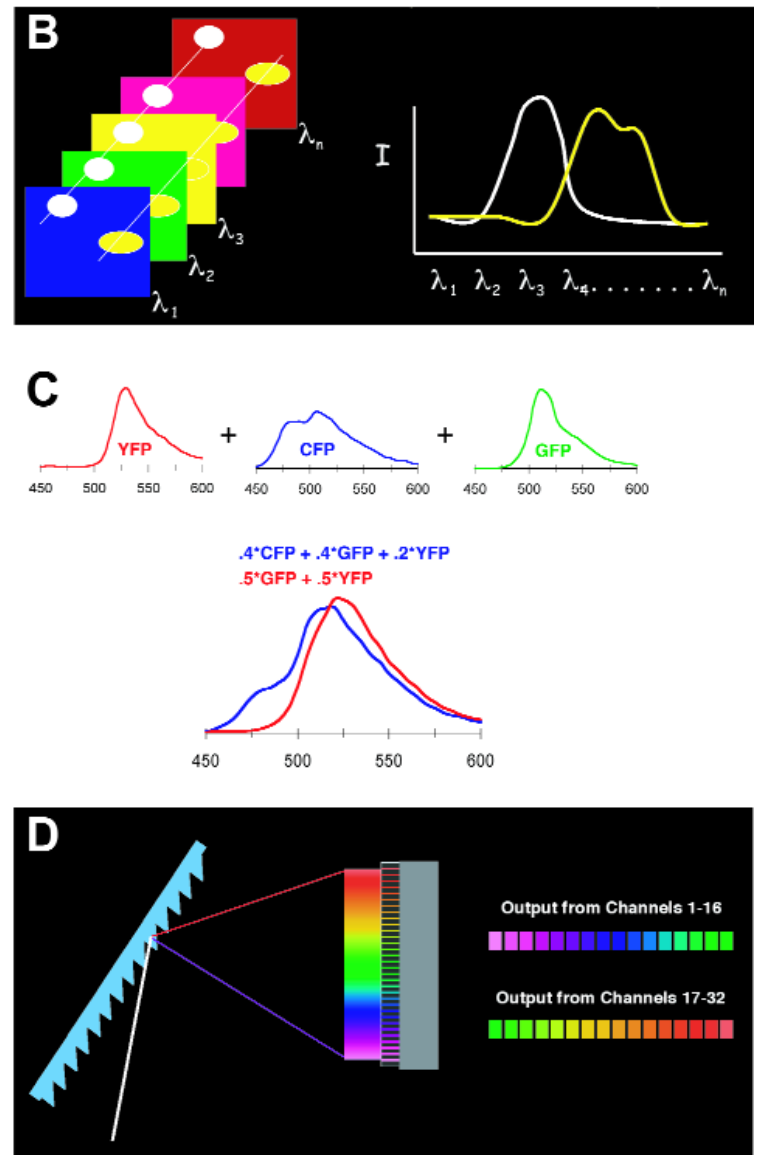

Figure 1. Separation of overlapping emission spectra. (a) Overlapping emission spectra of fluorescent protein variants. (b) Schematic representation of a lambda stack. The $x-y$ images are taken at several different wavelengths $\left(\lambda_{1}-\lambda_{n}\right)$ to create a 3-D stack. The average intensity (I) versus the wavelength $(\lambda)$ can be plotted to reveal the fluorescence emission spectrum of the dye in the image. (c) Spectrally mixed pixels combine emission spectra to yield an observed spectrum that is a linear sum of the components, weighted by their local concentration. This figure illustrates a few examples that produce relatively similar summed spectra that cannot be unraveled using filters. It is a linear algebra problem to deconvolve the weighting coefficients of the component spectra from the measured sum spectra. (d) Design of the META spectral detector (schematic). A grating is used to separate emission photons based on wavelength. A 32-channel PMT array collects the dispersed output in 10.7-nm bands across the visible spectrum.

\section{TRUE-COLOR SEPARATION THROUGH LINEAR ALGEBRA}

Three different methods can be used to classify pixels based on the spectral signature-principal components analysis (PCA), supervised classification (SCA), and linear unmixing. Two of these methods (SCA and linear unmixing) require prior knowledge of the reference spectra for a given dye, but only linear unmixing is appropriate for determining the co-localized probe identity and concentration of individual species in pixels that have a mixed contribution from different spectra. In fact, for spatially segregated probes with no spectral mixing, linear unmixing will give essentially the same results as PCA or SCA. In this sense, linear unmixing is the preferred method since the results are comparable to other methods for spatially segregated data, yet superior for generating unambiguous images of co-localized probes.

Below is an example chosen from recent work using an LCTF to collect spectral data from a mixed population of cells expressing CFP, GFP, and YFP simultaneously excited using two-photon excitation (2).

For a linearly mixed pixel containing CFP, GFP, or YFP, the measured spectrum, $\mathrm{S}$, of any pixel is expressed as

$$
\mathrm{S}(\lambda)=\mathrm{A}_{1} \mathrm{CFP}(\lambda)+\mathrm{A}_{2} \mathrm{GFP}(\lambda)+\mathrm{A}_{3} \mathrm{YFP}(\lambda)
$$

or more generally as

$$
\mathbf{S}(\lambda)=\sum \mathbf{A}_{\mathbf{i}} * \mathbf{R}_{\mathbf{i}}(\lambda) \text { or } \mathbf{S}=\mathbf{A}^{*} \mathbf{R},
$$

where $\mathbf{R}$ are the measured reference spectra. The spectra can be combined to create a measured summed spectrum (Figure 1c). It is a linear algebra problem to solve for the weighting matrix $\mathbf{A}$, and the solution is usually obtained with an inverse least square procedure that minimizes the difference between the measured and modeled spectrum. In this example, we

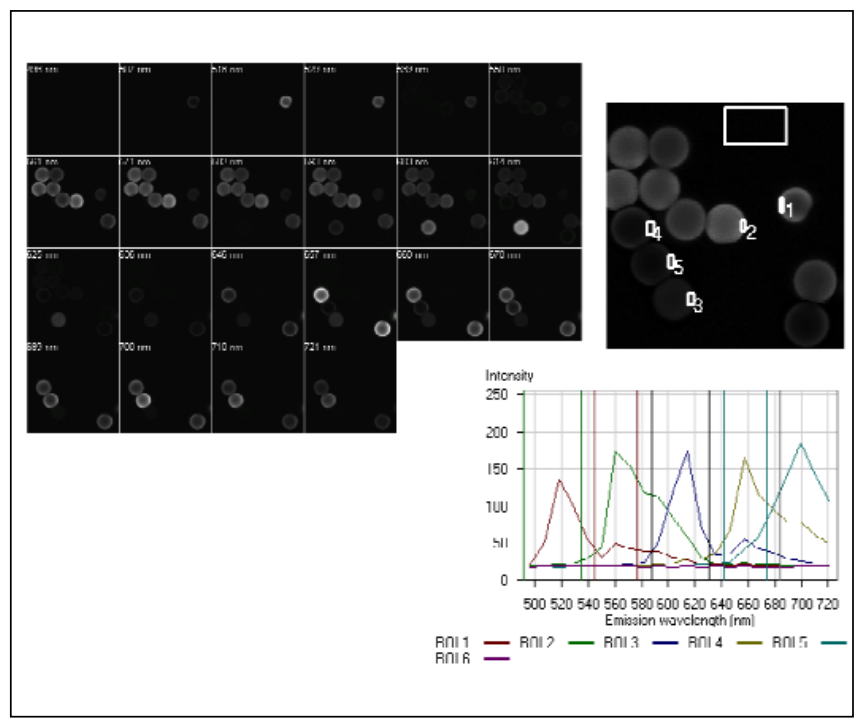

Figure 2. Emission spectra of fluorescent beads. A $512 \times 512 \times 22 x-y-\lambda-$ stack of images of fluorochrome-labeled beads (top left). Multiple excitation lines were used for dye excitation. ROIs are used to select pixels corresponding to individual beads (top right). Emission spectra of each of the regions of interest are shown on the right. 
used an algorithm that allowed a constrained unmixing to force the weights to sum to unity, making it easier to compare the separate images and threshold the data to classify pixels. The result of the linear unmixing is three images, each an image of the complete scene of the weighting coefficients, $\mathrm{A}_{\mathrm{i}}$, for each spectral component.

The results of a linear unmixing can be looked at in a couple of different ways. For example, one can threshold the data to locate pixels with a set amount of a probe. In effect, this is a classification, although with a loose criteria. Setting the bar to 0.6 , all pixels containing more than 0.6 of that spectral component are then classified as belonging to that probe. However, since some pixels may contain an equal value of all three fluorochromes (i.e., 0.3, 0.4, and 0.3), this method will produce some unclassified pixels.

A second visualization approach is to make what the re- mote sensing community terms abundance maps; pseudo-colored maps in which the pixel value of each image plane, or channel, which represents how much of a probe is present, is indicated by the color and intensity. Each channel that is produced is similar to the data obtained using different filter sets, and the channels can be overlaid to determine the overlapping distribution of each probe. This is perhaps the most generally applicable method of data presentation because the intensity of each probe is color coded.

\section{ADDING A NEW DIMENSION TO CONFOCAL AND TWO-PHOTON MICROSCOPY}

Until now, the methods described above required both specialized hardware to create lambda stacks and offline software

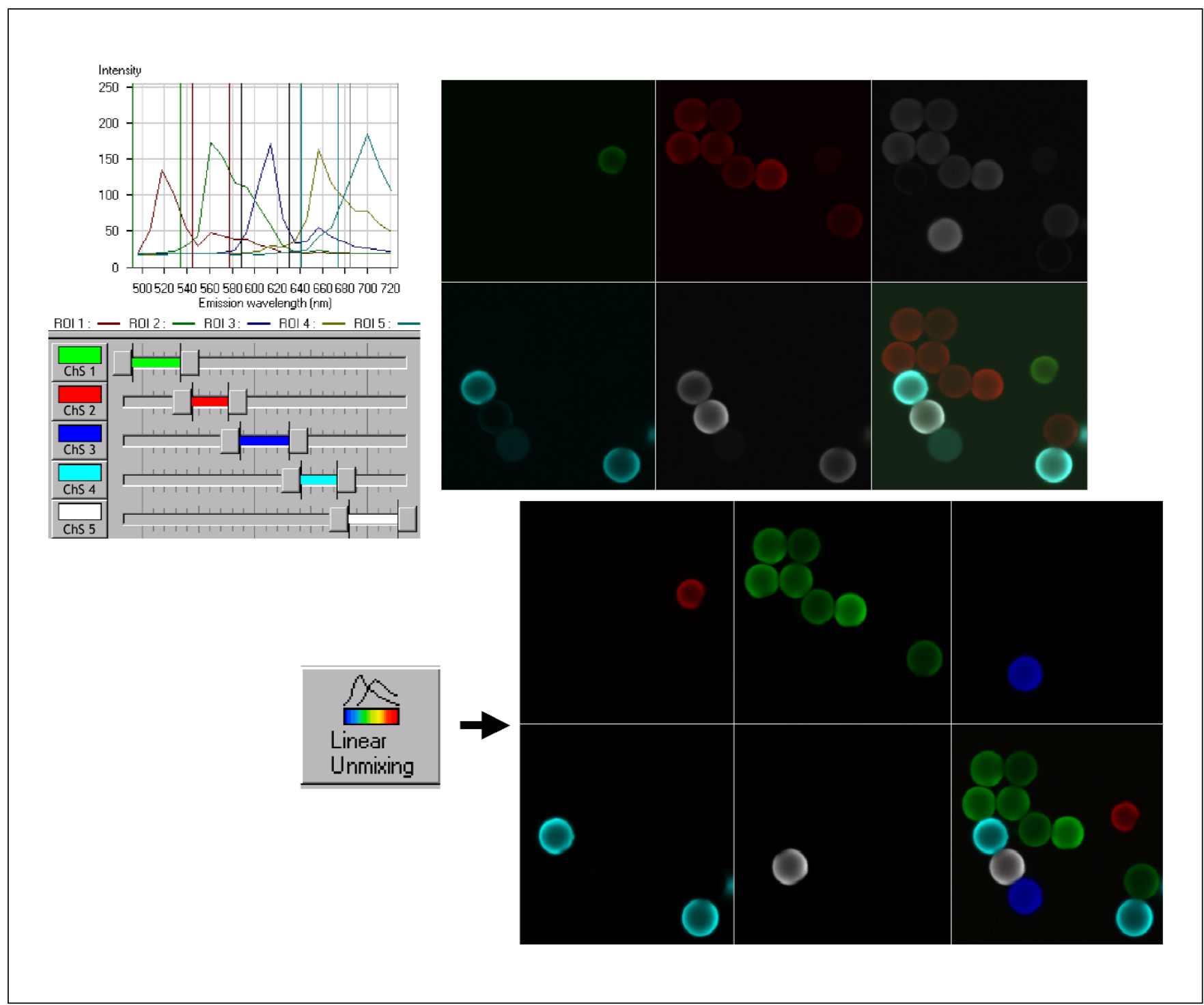

Figure 3. Output from a sample of mixed polystyrene beads. Beads are labeled with several different fluorochromes. The emission spectra of the dyes overlap and cannot be cleanly separated using multiple bandpass settings (top). Using linear unmixing algorithms, each dye can be clearly distinguished from one another (right). 
Table 1. Emission Fingerprinting Method Using Zeiss LSM 510 META

Three steps to clear emission separation:
(i) Acquire a lambda stack: record the whole emission
signal for every single pixel of an image.
(ii) Use the ROI function to determine the spectral
signature of a selected area of the scanned image.
(iii) Apply the linear unmixing algorithm on the lambda
stack using reference spectra a of all dyes in the
specimen.

Result:

Multi-fluorescence images with clear separated image channels representing one fluorochrome each.

aSpectral signatures containing just the emission signal of a single fluorochrome are defined as reference spectra.

packages, such as ENVI (2) to process the data. In addition, the spectral imagers discussed above are impractical for use in 3-D or 4-D analysis because image acquisition is too slow or insensitive, or the device cannot be used in a laser scanning microscope. What is needed is an easy-to-use system for lambda stack acquisition and linear unmixing for confocal and two-photon microscope users. The LSM 510 META (Carl Zeiss, Jena, Germany) combines all the capabilities of the existing LSM 510 with a fast, sensitive, and reliable spectral imager, including robust linear unmixing functions integrated into the standard LSM system software. The META hardware consists of a special grating as a dispersive element and a 32channel PMT array to collect photons across the visible spectrum (Figure 1d). Neither the grating nor the detector move; rather, the range of photons that are collected are specified by turning on or off individual detector channels. Thus, full flexibility of bandpass selection with solid reproducibility is possible. The META spectral imager replaces a single conventional PMT in the LSM 510 scan head and can be used in combination with the other on-board PMTs, all with adjustable pinholes, for maximum flexibility. In addition, already installed LSM 510 systems can be upgraded with the META detector, offering new technology to existing LSM 510 users.

This device expands the dimensions available to confocal and two-photon laser scanning microscope users. Using the META, spectral imaging can be combined with z-series collection, providing more information about the spatial relationship of molecules or structures within the cell or the organism. In addition, a full $512 \times 512$ lambda stack of data from all 32 channels (the full visible spectrum $350-700 \mathrm{~nm}$ ) can be acquired in $0.8 \mathrm{~s}$, and $\mathrm{x}-\mathrm{y}$ scans with fewer pixels or lambda stacks, including data from fewer channels, increase the speed considerably. For instance, data from eight channels can be scanned across a line $(1 \times 512$ pixels $)$ in as fast as $0.38 \mathrm{~ms}$. Therefore, it is possible to combine spectral imaging with time-lapse analysis or to image in multiple dimensions including three spatial dimensions, time and color, producing 5-D images.

\section{NEW DETECTION AND ANALYSIS FUNCTIONS OFFER MORE FLEXIBILITY}

Below are examples of the META package put to task. Consider a sample of mixed fluorescent beads (Figure 2). A lambda stack is generated over a series of wavelengths. In this example, $x-y$ images are taken using 22 of the 32 possible channels. To determine the spectra of each individual bead, a region of interest (ROI) can be specified, and the software

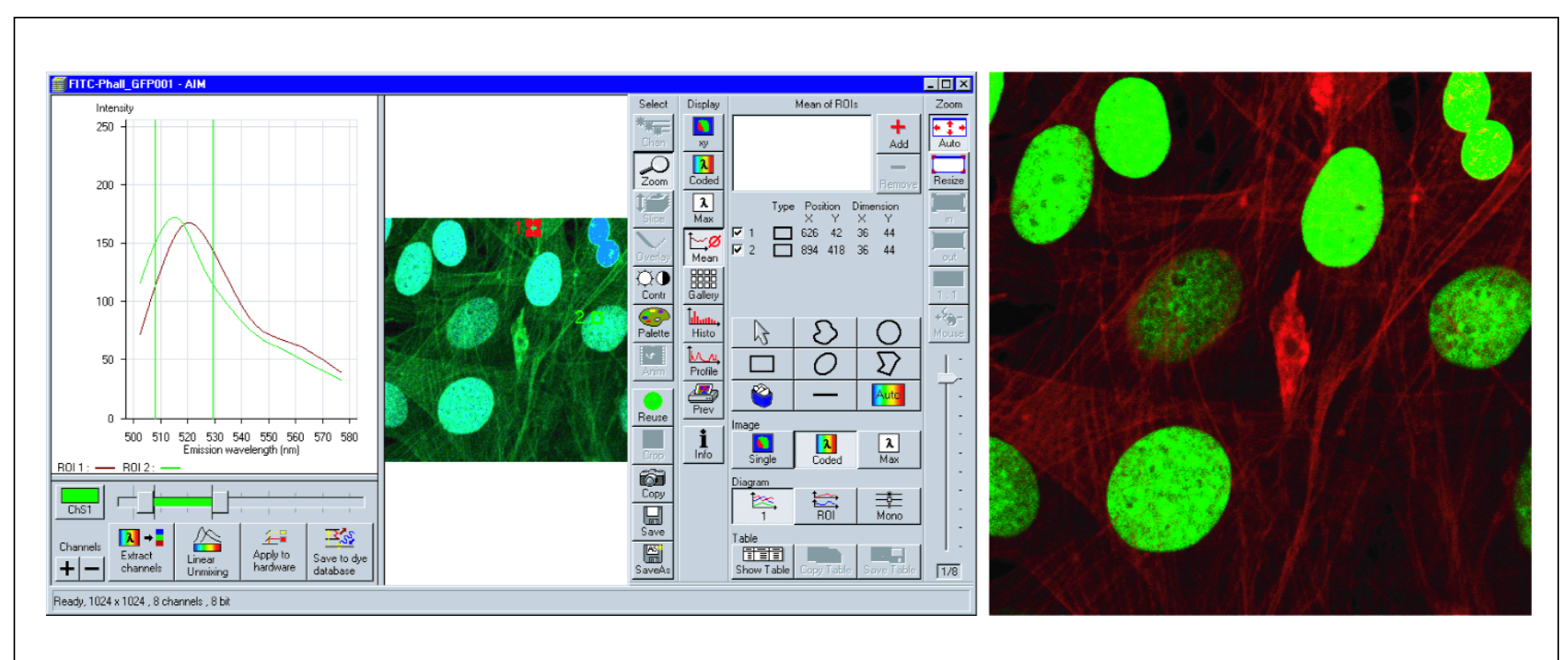

Figure 4. Linear unmixing of data from cells expressing two green fluorochromes-GFP (nucleus) and FITC (actin filaments). On the left is the original data in the LSM 510 META software interface, including the lambda-coded image (center) and the emission spectra from both dyes (left). The Lambda coded image matches the emission data with a pseudo-color that matches the wavelength range in the visible spectrum. Thus, both signals appear green, as there is only a 7$\mathrm{nm}$ separation between the two peaks. On the right is the image after linear unmixing where the GFP signal is shown in green and the FITC-Phalloidin signal is pseudo-colored red for maximum contrast. 
will display the mean intensity of all pixels within the ROI versus the wavelength.

Once the emission spectra are generated, several options are available to the user. Software sliders can be used to configure the bandpass settings of up to eight individual channels. For instance, one channel can be configured to show only the output that falls between 496 and $539 \mathrm{~nm}$, whereas another channel can be used to show emission signal from 636 to $720 \mathrm{~nm}$, and so on (Figure 3). In some cases, this added flexibility is sufficient to separate many fluorochromes; however, in the example shown above, the physical separation of emission spectra is not possible (Figure 3). In this instance, linear unmixing can be used to resolve the spectra in each pixel and assign different colors to the pixels corresponding to a particular dye. Reference spectra for each dye are collected using the ROI function, and pixels matching each spectral signature are pseudo-colored accordingly, resulting in an image where each dye is completely separated (Figure 3 ).

In the example above, there was considerable spectral cross-talk in the image, but each pixel contained emission signal from one single fluorochrome. As we discussed earlier, linear unmixing is also well suited for resolving spectral overlap in mixed pixels, that is, in pixels that have emission signal from more than one fluorochrome. For instance, consider a sample where the nucleus is labeled with GFP (using a histone 2B-GFP fusion; see Reference 2 for details) and actin filaments in the cytoplasm are labeled using fluorescein-conjugated Phalloidin (Molecular Probes, Eugene, OR, USA). These two fluorochromes have substantial spectral overlap, and the emission peaks are only $7 \mathrm{~nm}$ apart and are co-localized in some regions of the image. Despite both the spectral and spatial overlap, the linear unmixing algorithms are used to create an image in which the contribution from each fluorochrome is represented in a separate channel and shown here as distinct colors (Figure 4). Thus, the LSM 510 META with linear unmixing can be used to go beyond what can be achieved with physical separation and can be used to characterize samples containing co-localized, spectrally overlapping dyes.

The emission fingerprinting method described here (Table 1 ) is not only well suited for separating emission signals of multiple fluorophores, but it is also ideal for eliminating unwanted signals such as background or autofluorescence contribution from the image.

\section{CONCLUSION}

With the incorporation of the described multi-fluorescence imaging technique into an easy-to-use, commercially available system, the door is opened for far greater flexibility for many different applications in biomedical research. Not only can one use more probes in a single experiment but also dyes and fluorescent protein constructs that were once unusable because they did not fit standard filter sets, overlapped spectrally, or had a small Stokes shift can now be utilized to expand the tools available to researchers.

\section{REFERENCES}

1.Garini, Y., N. Katzir, D. Cabib, R.A. Buckwald, D.G. Soenksen, and Z. Malik. 1996. Spectral bio-imaging, p. 87-124. In X.F. Wang and B. Herman (Eds.). Fluorescence Imaging Spectroscopy and Microscopy. Wiley $\&$ Sons, New York.

2.Lansford, R., G. Bearman, and S.E. Fraser. 2001. Resolution of multiple green fluorescent protein color variants and dyes using two-photon microscopy. J. Biomed. Optics 6:311-318.

3.Levenson, R.M. and C.C. Hoyt. 2000. Spectral imaging and microscopy. Am. Lab. 32:26-34.

4.Malik, Z., D. Cabib, R.A. Buckwald, A. Talmi, Y. Garini, and S.G. Lipson. 1996. Fourier transform multipixel spectroscopy for quantitative cytology. J. Microsc. 182:133-140.

5.Nitschke, R., S. Wilhelm, R. Borlinghaus, J. Leipziger, R. Bindels, and R. Greger. 1997. A modified confocal laser scanning microscope allows fast ultraviolet ratio imaging of intracellular $\mathrm{Ca}^{2+}$ activity using Fura-2. Pflügers Arch.-Eur. J. Physiol. 433:653-663.

6.Schröck, E., S. du Manoir, T. Veldman, B. Schoell, J. Wienberg, M.A. Ferguson-Smith, Y. Ning, D.H. Ledbetter et al. 1996. Multicolor spectral karyotyping of human chromosomes. Science 273:494-497.

Address correspondence to Dr. Mary Dickinson, Biological Imaging Center, Beckman Institute, MC 139-74, California Institute of Technology, Pasadena, CA 91125, USA. e-mail: maryd@gg.caltech.edu

Suggestions for contributions to the Biolmaging feature are welcomed by its editor, Dr. Steve Paddock (paddock@facstaff.wisc.edu)

For reprints of this or any other article, contact Reprints@BioTechniques.com 